Гринкевич В. А.

\title{
ИССЛЕДОВАНИЕ ПРИМЕНЕНИЯ МЕТОДА ШТРАФНЫХ ФУНКЦИЙ С ЦЕЛЬЮ ВЫПОЛНЕНИЯ УСЛОВИЯ НЕСЖИМАЕМОСТИ НА ПРИМЕРЕ ОСАДКИ КЛИНА
}

При математическом (компьютерном) моделировании развитых процессов пластической деформации одной из серьезных проблем является учет условия постоянства объема (условия несжимаемости). Если при аналитическом методе решения краевой задачи удовлетворить условие несжимаемости относительно просто, то при численном решении, в частности, методом конечных элементов это условие можно удовлетворить лишь приближенно.

Наиболее распространенным способом решения краевой задачи является минимизация функционала Маркова-Германна [1]:

$$
I=\int_{v} T H d V+\int_{v} \sigma_{0} \varepsilon_{0} d V-\int_{S} F_{i} V_{i} d S \rightarrow \min ,
$$

где $T$ - интенсивность касательных напряжений; $H$ - интенсивность скоростей сдвиговых деформаций; $\sigma_{0}$ - среднее нормальное напряжение; $\varepsilon_{0}-$ средняя линейная скорость деформации; $F_{i}$ - компонента вектора поверхностных сил; $V_{i}-$ компонента вектора скорости течения.

В этом случае результатом решения являются как поле вектора скорости течения, так и, по сути, поле тензора напряжений. Это несомненное преимущество данного подхода. Однако здесь есть и недостаток. Разрешающая система алгебраических уравнений метода конечных элементов будет обладать не очень хорошими вычислительными свойствами: на главной диагонали будут нули в каждом третьем уравнении. Кроме того, среднее нормальное напряжение необходимо аппроксимировать и интегрировать иначе, чем скорости течения. Это требует усложнения соответствующих алгоритмов. В ряде случаев на практике это приводит к ухудшению результатов расчета по напряжениям.

Существует альтернативный подход. Это минимизация модифицированного функционала Лагранжа [2]:

$$
I=\int_{v} T H d V+\int_{v} t \varepsilon_{0}^{2} d V-\int_{S} F_{i} V_{i} d S \rightarrow \min ,
$$

где $t$ - положительная константа.

Второе слагаемое в правой части (2), в данном случае, представляет собой, так называемую, штрафную добавку, которая заставляет решение удовлетворять условию несжимаемости в смысле минимума квадрата его невязки. Принято считать, что чем больше штрафная константа, тем лучше решение удовлетворяет условию несжимаемости. Известно также, что чрезмерно большие константы ухудшают качество решения.

Заметим, что такой подход является предпочтительным в сравнении с предыдущим в смысле вычислительных свойств (2) - данный функционал является квадратичным; разрешающая система после конечно-элементной дискретизации - хорошо обусловленной. Недостатком данного подхода является необходимость вычисления напряжений после решения краевой задачи в скоростях течения.

В целом можно утверждать, что информация о применении метода штрафных функций при решении краевых задач пластичности носит преимущественно качественный характер. Следовательно, попытка получить количественную информацию на эту тему является актуальной. 
Для проведения вычислительного эксперимента использовался пакет программ FORGEOMD, разработанный автором данной статьи. Этот пакет позволяет решать двумерные (плоские и осесимметричные) краевые задачи пластической деформации. Для определения поля скоростей используется выражение (2). Для учета скоростного и деформационного упрочнения применяется метод гидродинамических приближений. Температурное поле определяется из решения нелинейного уравнения теплопроводности методом Галеркина. Для конечно-элементной дискретизации используется изопараметрический квадратичный элемент сирендипова семейства.

Целью вычислительного эксперимента являлось определение количественного влияния величины штрафной константы на скорость неравномерной объемной деформации. Для исключения влияния контактного трения и эффектов упрочнения была принята деформация без контактного трения линейно-вязкой (ньютоновской) среды. Неравномерность деформации обеспечивалась геометрией заготовки - моделировалась осадка клина. Краевая задача решалась в безразмерном виде. Размеры половины поперечного сечения таковы: ширина нижней грани -5 , верхней -4 , высота -4 . Скорость опускания верхнего бойка постоянна и равна -1 . Условная вязкость среды по всему поперечному сечению заготовки была постоянна и равнялась 1.

Поскольку не ставилась задача получить близкое к точному решение, сетка состояла из 4-х конечных элементов. Исходная сетка представлена на рис. 1.

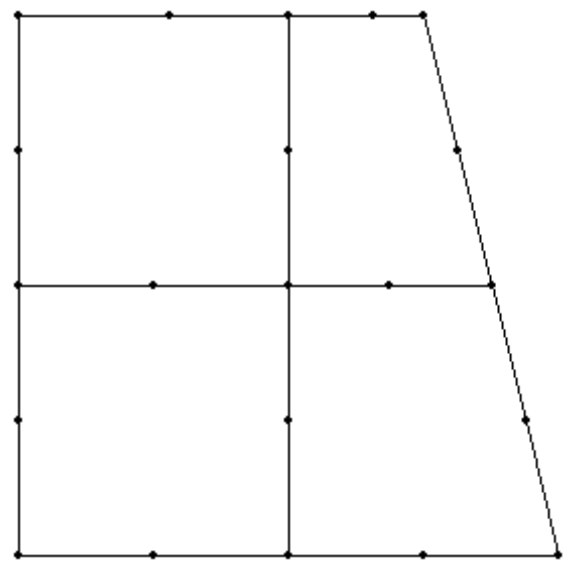

Рис. 1. Исходная конечно-элементная сетка

Расчеты производились для следующих значений штрафной константы: $0.1,1,10,50$, 100, 1000 и 10000. В качестве основного результата расчетов была принята скорость относительного изменения объема $\varepsilon_{0}$ в узловых точках сетки. Среднее значение этой величины вычислялась, как среднее арифметическое во всех узловых точках. Результаты расчетов приведены в табл. 1.

Таблица 1

Результаты расчетов

\begin{tabular}{|c|c|c|c|c|c|c|c|}
\hline $\mathrm{t}$ & 0.1 & 1 & 10 & 50 & 100 & 1000 & 10000 \\
\hline$\varepsilon_{0 \text { cp. }} \%$ & -23.6 & -16.3 & -3.5 & -0.1 & 0.4 & 0.8 & 0.9 \\
\hline
\end{tabular}

Типичная искаженная конечно-элементная сетка представлена на рис. 2.

Следует отметить, что при отсутствии контактного трения искривление боковой поверхности заготовки обусловлено подпором со стороны боковой грани - скольжение на верхней грани несколько затруднено. На рис. 3 представлено поле скоростей для данного варианта расчета. Формоизменение на рис. 1, а также на рис. 2 и 3 показано в разном масштабе. 


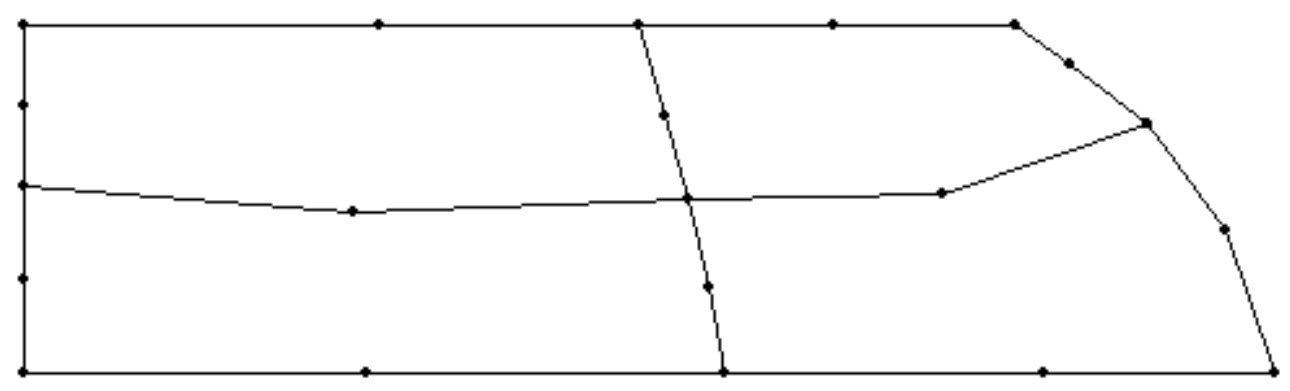

Рис. 2. Формоизменение при осадке на $20 \%$ при $\mathrm{t}=50$

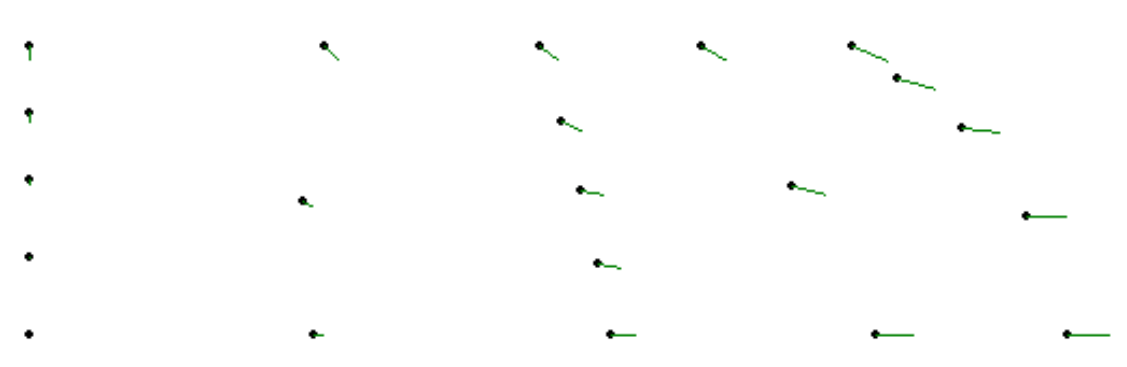

Рис. 3. Поле скоростей при осадке на 20 \% при $\mathrm{t}=50$

Необходимо отметить, что при решении конкретных краевых задач данное (опорное) поле скоростей уточняется при помощи итеративных процедур, чаще всего по методу гидродинамических приближений. Кроме того, на рис. 2 видно, что условие несжимаемости для серединных узлов центральных конечных элементов выполняется приближенно. По-видимому, это происходит из-за вычислительных свойств сирендипова квадратичного элемента. В целом следует понимать, что метод штрафных функций в данной трактовке приводит к довольно точному удовлетворению условию несжимаемости во всем объеме и к приближенному выполнению в отдельных точках очага деформации. Также на рис. 2 и 3 видно, что распределение горизонтальных скоростей на боковой грани заготовки представляет собой квадратичную параболу, что соответствует свойствам ньютоновской среды.

На рис. 4 представлено распределение величины $\varepsilon_{0}$ на нижней грани заготовки при различных значениях штрафной константы.

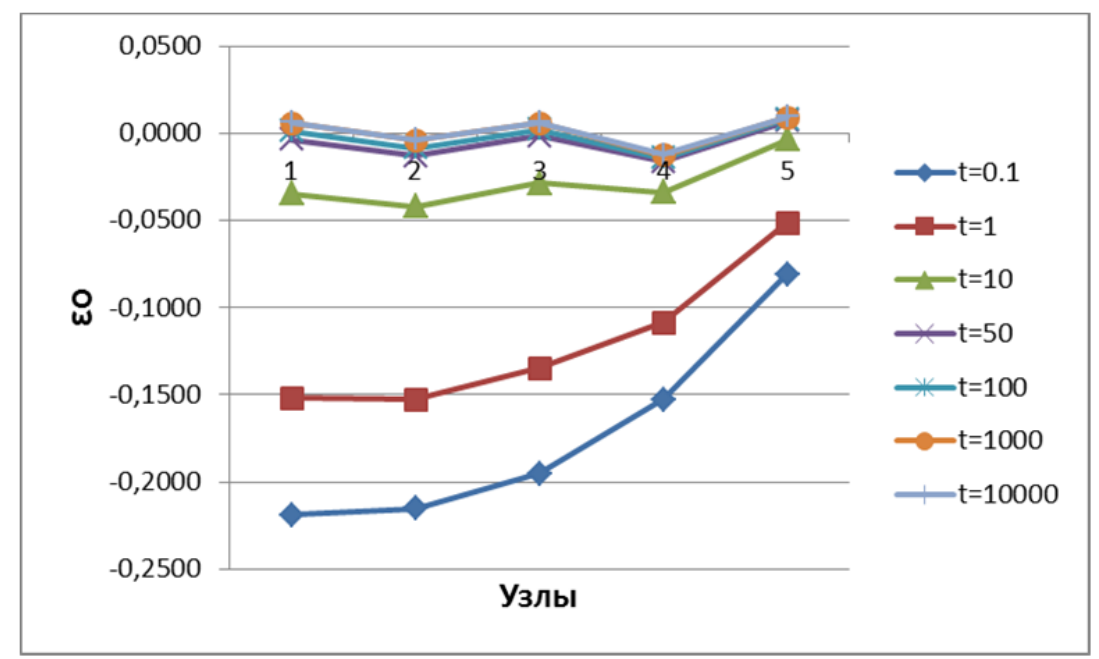

Рис. 4. Распределение величины $\varepsilon_{0}$ на нижней грани заготовки 
Прокомментируем результаты, приведенные в табл. 1. Очевидно, что значения штрафной константы в интервале от 0.1 до 1 приводят к весьма значительной средней скорости изменения (потере) объема, что не соответствует гипотезе постоянства объема. С другой стороны, штрафные константы, лежащие в интервале от 100 до 10000, дают неправдоподобные результаты - средняя скорость изменения объема положительна (хотя и незначительна), что противоречит современным представлениям о процессе пластической деформации. Интерес представляет интервал от 10 до 50, внутри которого и лежит реальная средняя скорость изменения объема. Можно предположить, что оптимальное, то есть дающее близкое к экспериментальным данным, изменение (небольшое уменьшение) объема, будет при штрафной константе в интервале от 35 до 40.

Далее проанализируем графики, приведенные на рис. 4. Для небольших (0.1-1) значений штрафной константы наблюдается плавное увеличение скорости изменения объема от центра к периферии образца. Начиная со значения 10 распределение существенно выравнивается. Кроме того, наблюдается осцилляция в серединных узлах конечно-элементной сетки. Это можно объяснить некорректностью требования несжимаемости во всех восьми узлах конечного элемента, поскольку распределение скорости деформаций в таком элементе билинейное. Из графиков видно, что распределение в угловых узлах монотонно возрастающее. Также видно, что, начиная со значений константы 50, распределение качественно меняется, то есть оно становится почти постоянным и положительным, что не соответствует физике пластической деформации.

В целом, можно сказать, что при рациональном задании штрафной константы можно добиться неплохого приближения к условию несжимаемости. Кроме того, можно приблизиться к простому и достаточно точному вычислению напряженного состояния, но это требует дополнительных исследований.

\section{ВЫВОДЫ}

Проведено исследование влияния значения штрафной константы на скорость относительного изменения объема при деформации линейно-вязкой (ньютоновской) среды при осадке клина.

Определен рациональный интервал значений штрафной константы.

\section{СПИСОК ИСПОЛЬЗОВАННОЙ ЛИТЕРАТУРЫ}

1. Данченко В.Н., Миленин А.А., Кузьменко В.И., Гринкевич В.А.. Компьютерное моделирование процессов обработки металлов давлением. Дніпропетровськ: Системні технології. 2005. 488 с.

2. Балакин В.Ф., Фень Г.А., Кузьменко В.И. и др. Гидропрессование труб. Киев: Техніка. 1986. 136 с.

\section{REFERENCES}

1. Danchenko V.N., Milenin A.A., Kuzmenko V.I., Grinkevich V.A. Computer simulation of metal forming processes. Dnepropetrovsk: System technologies. 2005, 488 p.

2. Balakin V.F., Fen G.A., Kuzmenko V.I. et al. Hydraulic pressing of pipes. Kyiv: Tekhníka. 1986, 136 p.

Гринкевич В. А. - д-р техн. наук, проф. НМетАУ.

НМетАУ - Национальная металлургическая академия Украины, г. Днепр.

E-mail: grinkevich@metal-forming.org. 